\title{
An early Medical Research Council controlled trial of vitamins for preventing infection
}

\author{
Irvine Loudon
}

J R Soc Med 2007; 100:195-198

Additional material for this article is available from the James Lind Library website [www.jameslindlibrary.org], where this paper was previously published.

\section{BACKGROUND}

The background to the controlled trial of vitamin A to prevent infection after childbirth reported by Green et al. in $1931^{1}$ was as follows. As a result of experiments on animals in the mid to late 1920 s, the senior co-author of the 1931 report_-Sir Edward Mellanby, Professor of Pharmacology in Sheffield - believed that an important function of vitamin A was to 'raise resistance-either local or general or both - to bacterial infection.' ${ }^{2}$ Accordingly, he decided to administer vitamin A to women with puerperal septicaemia in the Jessop Hospital, Sheffield, to see whether it had 'any specific action in human infection.'

Mellanby and Green reported the results of this study in 1929.2 They explained that they had chosen to study puerperal septicaemia for two reasons. First, it was argued, many women had sub-optimal stores of vitamin $\mathrm{A}$ in the liver but 'the pregnant woman is in still greater danger of a deficiency' [of vitamin A] because she had to supply the vitamin to her baby 'whose metabolic rate is relatively much greater than that of the adult.' The second reason was 'the difficulty in clinical work of assessing the value of a remedy in diseases which often have a low mortality rate whatever the treatment given.' In the 1920s the fatality rate of puerperal septicaemia (proven by a positive blood culture) was over 90\%. All the women described in the Mellanby and Green paper had had positive blood cultures. Women with negative blood cultures were excluded, even if, on clinical grounds, they might be thought to be cases of puerperal septicaemia. $^{2}$

The results of this first use of large doses of vitamin A as an anti-infective agent were astonishing, and are shown in Table $1 .^{2}$

The five cases of puerperal septicaemia in the first three months of 1929 were all treated with vitamin A. All five made a complete recovery and left the hospital restored to normal good health.

The Mill House, Wantage, Oxon OX12 9EH, UK

E-mail: irvine.loudon@green.ox.ac.uk
Mellanby and Green were cautious in interpreting these observations, however, noting that, although impressive, the results were 'too few in number to allow the deduction that this form of treatment is specific in its nature for septicaemia.' They saw that 'clinical trials should be made,' and judged that because vitamin A 'must also be of inestimable value in its prophylactic effect,' the vitamin should be given for several weeks before delivery, to build up stores of vitamin $\mathrm{A}$ in the liver of expectant mothers. ${ }^{2}$

\section{THE CONTROLLED TRIAL}

The results of this study proposed in the 1929 paper were reported two years later by Green et al. ${ }^{1}$ The 1931 paper is one of the earliest reports of a controlled clinical trial supported by the British Medical Research Council. The investigation began in Sheffield in 1929. Women who had attended antenatal clinics at the Jessop Hospital and a local municipal clinic were allocated to receive or not receive supplements of vitamins A and D as follows:

'The cases were in no way selected; the first patient was given the preparation and the next due for delivery about the same time was indexed as a control. The vitamin and control groups were thus equally distributed in point of time; seasonal or epidemic or contagious influences predisposing to infection therefore tended to be equal in the two groups. It was considered that this method would give more uniform conditions than treating random groups of ante-natal patients with the preparation and considering all the remaining untreated antenatal cases as controls.'

The alternation scheme appears to have been strictly observed because there are equal numbers in the two comparison groups generated, and there no evidence of (differential) dropouts after allocation.

The report discusses possible failure to comply with the vitamin prescriptions, and the implications. Although it does not address control of observer bias, a later commentary on the study ${ }^{3}$ stated that the doctors and midwives who attended the deliveries of these women were not informed which patients had received vitamin supplements and which had not. The observed differences in outcome were tested statistically, and analyses were done 


\begin{tabular}{llll}
\hline & \multicolumn{2}{c}{ Prior to Vitamin A Treatment } & \multirow{2}{*}{ Vitamin A Treatment } \\
\cline { 2 - 3 } Year & 1927 & 1928 & \multirow{2}{*}{1929 (3 months) } \\
\hline Number of cases & 8 & 16 & 5 \\
Number of recoveries & 2 & 0 & 5 \\
\hline
\end{tabular}

to assess how sensitive these were to different ways of classifying the outcomes.

The context of the 1929 and 1931 reports is important. From about 1910 to the early 1930s the role and biochemical structure of the vitamins was the subject of a very large amount of research, carried out in the hope that the nature and prevention of many previously intractable diseases could be discovered. Thus the structure of vitamin A was first described in 1931, the year in which this paper was published. It is also important to remember that this paper was concerned with preventing puerperal sepsis, a disease which was a major problem in obstetrics, and remained so until the introduction of the sulphonamides in 1937. In 1931, any measure apart from improved antisepsis and asepsis which claimed to reduce the incidence and mortality of this dreadful disease should have attracted at least some attention.

Although, for its time, this was a well organized trial, it had two features which I believe diminished its importance. The source of the vitamin A given to the treated group was a vitamin preparation called Radiostoleum, manufactured by British Drug Houses, which donated supplies of this drug for this investigation. Radiostoleum contained both vitamin A and vitamin D in a neutral oil. The authors mentioned this briefly, saying they thought that 'on experimental grounds we are inclined to believe that vitamin D has little anti-infective action.' It would surely have been better to have given vitamin A alone, but it may be that no such preparation was easily available.

The second problem is more important. When it came to comparing the incidence of puerperal infection in the treated and the control group, the authors used what they called the 'BMA [British Medical Association] standard for puerperal morbidity' because this had been in use for statistical purposes in the Jessop Hospital, Sheffield for some years, and would thus facilitate comparisons over time. The BMA standard defined puerperal infection as 'any [postnatal] patient who suffered a rise in temperature to $100 \mathrm{~F}$ or over, on at least two occasions between the end of the first and the end of the eighth day after delivery, regardless of the cause of the fever' (my italics). There was a reason for this which is not mentioned in the paper but is worth explaining.
In the late nineteenth century it was recognized that while there were profuse published records of mortality there were no official records of morbidity. This led to the Notification of Diseases Act, which was introduced in 1899. The Act listed a number of infective diseases which had to be notified by the doctor in attendance to the public health authorities. One was puerperal fever.

There is no doubt that puerperal fever was a distinct, definable disease. It consisted of a bacterial infection of the genital tract occurring in the postnatal period. The infection might be confined to the uterus, but it often spread to cause peritonitis or septicaemia or both. A large majority of the seriously ill and fatal cases were due to Streptococcus pyogenes, but a few were due to Staphylococcus aureus and some milder cases were due to Bacterium coli. ${ }^{4}$

In the early years of the nineteenth century, when nosography and nosology were based on postmortem appearances, there were numerous synonyms for puerperal fever. We can forget these, except to say that the original term 'puerperal fever' managed to survive until the $1890 \mathrm{~s}$ as the title of first choice. Following the discoveries of bacteriology, however, various authorities began to protest that puerperal fever was not a fever. Fevers were always due to one specific organism. In his large and influential work, Puerperal Infection, Arnold Lea argued that the use of 'fever' was wrong. ${ }^{5}$ He recommended that 'puerperal fever' should be replaced by 'puerperal infection' which would have been ideal but was never accepted. Before this, however, others had suggested the puerperal fever should be renamed as 'puerperal sepsis', and that was accepted by the authorities who drew up the list of notifiable disease. It was, incidentally, the only notifiable disease which presented this kind of nosological problem.

Unfortunately, the term 'puerperal sepsis' caused confusion. Many doctors, believing that 'puerperal sepsis' meant 'puerperal septicaemia', assumed that they were only required to notify cases who were severely ill with clinical signs of septicaemia. Since many fatal cases of puerperal fever died without showing such signs, they died without having been notified. Fothergill (a Manchester obstetrician) noted in 1920 that in 93 counties and county boroughs there were 310 deaths from puerperal fever/sepsis. Because the fatality rate of puerperal fever was known to be in the 
region of $20 \%$, these 310 deaths should have generated around 1,500 notifications. In fact, there were only 243 notifications, giving an impossible fatality rate of 127 per cent. As he dryly commented: 'twenty years of compulsion has only secured comic figures.'

In 1926, recognizing the muddle caused by the term 'puerperal sepsis', the Ministry of Health went to the other extreme by renaming puerperal fever as 'puerperal pyrexia', which was defined as 'any fever of $100 \mathrm{~F}$ or over on at least two occasions within twenty-one days after childbirth or miscarriage regardless of the cause of the fever. ${ }^{7}$ It was not a happy decision. Pyrexia, of course, means a raised body temperature, regardless of cause. By removing the need to notify the cause of fever in the puerperium, the introduction of the term 'puerperal pyrexia' replaced one set of figures unreliable because of under-notification with another set just as unreliable because it led to over-notification. For this reason, the published notifications of puerperal fever between 1899 and the 1930 s are so flawed as to be valueless. ${ }^{4}$

Now the title of this paper-'Diet as a Prophylactic Agent against Puerperal Sepsis' - states clearly that it has measured the incidence of 'sepsis' in a group of 550 postnatal women. ${ }^{1}$ Yet the list of diseases included under this definition (Table 1 on page 596) included cystitis (nine cases), mastitis (seven cases), influenza (one case), gonorrhoea (one case), cervicitis (one case) and septic perineum (two cases). There were also seven cases of endometritis and one case of streptococcal septicaemia, and it is only these eight cases (and possibly the cases of cervicitis and septic perineum) that could be considered as cases of true puerperal fever, as defined above. The title of this paper should have been 'Diet as a Prophylactic Agent against Pyrexia in the Puerperium' (my italics again). The change from 'puerperal sepsis' to 'puerperal pyrexia' for the purposes of notification is not mentioned in the paper, but it is clear that if Mellanby had not included all cases of puerperal morbidity such as mastitis, cystitis and gonorrhoea, he would not have had enough cases to achieve a statistically significant result.

What the study seems to show is that dietary supplements of vitamins A and D reduced infective disease morbidity in general, which happened to occur in the puerperium, by an amount which was statistically significant. This is very different from showing that vitamin A reduced morbidity specifically due to puerperal fever/sepsis, defined as a bacterial infection of the genital tract which was either confined to the genital tract (the uterus and fallopian tubes) or spread from the genital tract to cause peritonitis or septicaemia or both. In the 1920s and early 1930s middle and upper class women were delivered at home or in private nursing homes. The participants in this trial were likely to have been predominantly working class women living in an industrial city during the great depression. This suggests that many of the patients may well have suffered from malnutrition, increasing one's readiness to accept this conclusion.

\section{IMPACT ON PRACTICE AND RESEARCH}

What impact did this paper have in the world of specialist and general practitioner obstetricians and midwives?

It is a very difficult question. The paper may have led doctors and midwives to learn, by word of mouth, the importance of dietary supplements in general and of vitamin A in particular as part of antenatal care. The only positive evidence comes in a famous book, Antenatal and Postnatal Care, by Francis J Browne, which was first published in $1935^{3}$ and went into 12 editions (the latest being in 1970). It came out at a time when antenatal care was beginning to become more widespread. It was probably the text on obstetrics most often read by generations of medical students. On pages 61 and 62 in the first edition there is a good account of the study. It is not presented as an important advance, however, but rather as part of a much wider general discussion of the importance of nutrition in pregnancy: and no-one could quarrel with that.

A search of Index Medicus and the indices of the British Medical Journal and the Lancet in the early 1930s reveal an enormous number of publications on the vitamins in general, including vitamin A, but I found only two papers which dealt with the role of vitamin $A$ and resistance to infection. In neither paper is Mellanby's report mentioned. From 1937 onwards, any slight influence this paper may have had is likely to have been drowned by the much more dramatic results of using first of all the sulphonamides in the treatment of puerperal fever, ${ }^{8}$ and later by the use of penicillin. Another factor is that the 1929 paper, which is the subject of the preface to this commentary, is simple, clear, and startling. The 1931 paper (the main subject of this commentary) is much more muddled and difficult to read, which may have reduced its impact.

What impact did the design of the study - the use of alternation to generate comparable groups and (apparently) blind outcome assessment - have on the design of subsequent clinical trials supported by the Medical Research Council?

One might expect it to have been influential. The fifth of the five authors of the study report was Sir Edward Mellanby (1884-1955), a leading expert on the role of nutrition in general and the vitamins in particular. Two years after the study was reported, Mellanby left the Chair of Pharmacology in Sheffield to become Secretary of the Medical Research Council. It is somewhat surprising, therefore, that during the decade following his appointment, the Medical Research Council made little use ${ }^{9}$ of the 
elements of controlled trial design (alternation and blind outcome assessment) that Mellanby and his colleagues in Sheffield had used in the late 1920s.

\section{REFERENCES}

1 Green HN, Pindar D, Davis G, Mellanby E. Diet as a prophylactic agent against puerperal sepsis, with special reference to Vitamin $\mathrm{A}$ as an antiinfective agent. BMJ 1931;2:595-8

2 Mellanby E, Green HE. Vitamin A as an anti-infective agent. Its use in the treatment of puerperal septicaemia. BMJ 1929;1:984-6

3 Browne FJ. Antenatal and Postnatal Care. London: J \& A Churchill, 1935
4 Loudon I. The Tragedy of Childbed Fever. Oxford: Oxford University Press, 2000

5 Lea AWW. Puerperal Infection. Oxford: Oxford Medical Publications, 1910

6 Fothergill WE. Puerperal pelvic infection. BMJ 1924;1:773-4

7 Ministry of Health. Circular no. 722. MoH, 1926. Cited in Loudon I. Death in Childbirth. Oxford: Oxford University Press, 1992:51-2

8 Loudon I. The use of historical controls and concurrent controls to assess the effects of sulphonamides, 1936-1945. The James Lind Library (www.jameslindlibrary.org), 2002

9 Toth B. Clinical Trials in British Medicine 1858-1948, with Special Reference to the Development of the Randomized Controlled Trial. PhD thesis, University of Bristol, 1998 